Marquette University
e-Publications@Marquette

Electrical and Computer Engineering Faculty Research and Publications

Electrical and Computer Engineering,

\title{
2-2014
}

\section{Speech Enhancement Using Bayesian Estimators of the Perceptually-Motivated Short-Time Spectral Amplitude (STSA) with Chi Speech Priors}

\author{
Marek B. Trawicki \\ Marquette University, marek.trawicki@marquette.edu \\ Michael T. Johnson \\ Marquette University, michael.johnson@marquette.edu
}

Follow this and additional works at: https://epublications.marquette.edu/electric_fac

Part of the Computer Engineering Commons, and the Electrical and Computer Engineering Commons

\section{Recommended Citation}

Trawicki, Marek B. and Johnson, Michael T., "Speech Enhancement Using Bayesian Estimators of the Perceptually-Motivated Short-Time Spectral Amplitude (STSA) with Chi Speech Priors" (2014). Electrical and Computer Engineering Faculty Research and Publications. 49.

https://epublications.marquette.edu/electric_fac/49 
Marquette University

e-Publications@Marquette

\section{Electrical and Computer Engineering Faculty Research and Publications/College of Engineering}

This paper is NOT THE PUBLISHED VERSION; but the author's final, peer-reviewed manuscript. The published version may be accessed by following the link in the citation below.

Speech Communication, Vol. 57 (February 2014): 101-113. DOI. This article is (C Elsevier and permission has been granted for this version to appear in e-Publications@Marquette. Elsevier does not grant permission for this article to be further copied/distributed or hosted elsewhere without the express permission from Elsevier.

\section{Speech Enhancement Using Bayesian Estimators of The Perceptually Motivated Short-Time Spectral Amplitude (STSA) With Chi Speech Priors}

\section{Marek B. Trawicki}

Marquette University, Department of Electrical and Computer Engineering, Speech and Signal Processing Laboratory, Milwaukee, WI

Michael T. Johnson

Marquette University, Department of Electrical and Computer Engineering, Speech and Signal Processing Laboratory, Milwaukee, WI

\section{Abstract}

In this paper, the authors propose new perceptually-motivated Weighted Euclidean (WE) and Weighted Cosh (WCOSH) estimators that utilize more appropriate Chi statistical models for the speech prior with Gaussian 
statistical models for the noise likelihood. Whereas the perceptually-motivated WE and WCOSH cost functions emphasized spectral valleys rather than spectral peaks (formants) and indirectly accounted for auditory masking effects, the incorporation of the Chi distribution statistical models demonstrated distinct improvement over the Rayleigh statistical models for the speech prior. The estimators incorporate both weighting law and shape parameters on the cost functions and distributions. Performance is evaluated in terms of the Segmental Signalto-Noise Ratio (SSNR), Perceptual Evaluation of Speech Quality (PESQ), and Signal-to-Noise Ratio (SNR) Loss objective quality measures to determine the amount of noise reduction along with overall speech quality and speech intelligibility improvement. Based on experimental results across three different input SNRs and eight unique noises along with various weighting law and shape parameters, the two general, less-complicated, closed-form derived solution estimators of WE and WCOSH with Chi speech priors provide significant gains in noise reduction and noticeable gains in overall speech quality and speech intelligibility improvements over the baseline WE and WCOSH with the standard Rayleigh speech priors. Overall, the goal of the work is to capitalize on the mutual benefits of the WE and WCOSH cost functions and Chi distributions for the speech prior to improvement enhancement.

\section{Keywords}

Speech enhancement, Probability, Amplitude estimation, Phase estimation, Parameter estimation

\section{Introduction}

Speech enhancement systems concern themselves with reducing the corrupting background noise in the noisy signal (Loizou, 2007). The most common approach is to perform statistical estimation: minimize the Bayes Risk of the squared-error of the spectral amplitude cost function, which leads to the subsequent and traditional Ephraim and Malah Minimum Mean-Square Error (MMSE) short-time spectral amplitude (STSA) estimator Ephraim and Malah, 1984. Based on the effectiveness of that STSA estimator, researchers began to modify the squared-error of the spectral amplitude cost function to utilize more subjectively meaningful cost functions. Ephraim and Malah (Ephraim and Malah, 1985) also developed and implemented the MMSE logspectral amplitude (LSA) estimator that minimizes the squared-error of the log-spectral amplitude, which is a more subjectively meaningful cost function that correlates well with human perception. From the STSA and LSA cost functions, Loizou (2005) constructed several perceptually-motivated spectral amplitude cost functions that emphasized spectral valleys rather than spectral peaks (formants) and indirectly accounted for auditory masking effects. Specifically, the Weighted Euclidean (WE) and Weighted Cosh (WCOSH) Bayesian estimators, which applied a weighting law parameter to the STSA cost function, had the best performances for reducing residual noise and producing better speech quality. In each of those corresponding spectral amplitude, log-spectral amplitude, and perceptually-motivated spectral amplitude estimators, the cost functions employed Rayleigh distributions for the statistical models of the speech priors and noise likelihoods.

Eventually, researchers began to exploit alternative and more accurate statistical modeling assumptions to the Rayleigh distribution for both the speech prior and noise likelihood using the STSA cost function. Andrianakis and White (2009) continued with the MMSE spectral amplitude estimators using the Gamma distribution but introduced the Chi distribution for modeling the speech priors. The Chi speech prior contains a shaping parameter that was varied to determine its effect on the quality of enhanced speech. From the results, the performance of the estimators was dependent on the shaping parameter, which controlled the trade-off between the level of residual noise and musical tones. As a generalization to the Ephraim and Malah's MMSE STSA and LSA estimators along with Andrianakis and White's Chi distribution speech priors, Breithaupt et al. (2008) developed a MMSE STSA estimator that uses both a variable compression function in the error criterion and the Chi distribution as a prior model. The resulting two parameters provide for the reduction of musical noise, speech distortion, and noise distortion. Through the incorporation of Chi distribution statistical models for 
the speech prior, the squared-error cost functions demonstrated distinct improvement over the Rayleigh statistical models.

Despite the success of the spectral amplitude, log-spectral amplitude, and perceptually-motivated cost functions with Rayleigh statistical models and spectral amplitude cost functions with Chi distributions for the speech priors, there has not been any work to capitalize on their mutual benefits for speech enhancement. Specifically, the improved statistical models for the speech prior have only been incorporated with the original MMSE STSA estimator, not with the spectral amplitude perceptually-motivated spectral amplitude (WE and WCOSH) cost functions. The fundamental purpose is to determine the effectiveness that more accurate speech priors would have on improved cost functions for noise reduction. Instead of utilizing the Rayleigh distributions for the speech prior, the Chi distribution is employed in this work since it leads to more general, less complicated, and more closed-form estimator solutions. For specific values of the shaping parameter, Chi distribution is equivalent to the half-Gaussian and Rayleigh distribution as special cases. Therefore, the focus of this work is to use the MMSE WE and WCOSH estimators with the Chi spectral speech prior distribution (Johnson et al., 1994) for reducing the background noise along with improving overall speech quality and speech intelligibility.

The remainder of this paper is organized into the following sections: system and statistical models (Section 2), perceptually-motivated cost functions with Chi speech priors (Section 3), experiments and results (Section 4), and conclusion (Section 5).

\section{System and statistical models}

In the time domain, the single channel additive noise model is given as

(1) $y(t)=s(t)+d(t)$

where $s(t), d(t)$, and $y(t)$ represent the clean, noise, and noisy signals. By taking the short-time Fourier Transform, (1) can be written in the frequency domain as

(2)

$$
\begin{gathered}
Y(l, k)=S(l, k)+D(l, k) \\
R(l, k) e^{j \vartheta(l, k)}=X(l, k) e^{j \alpha(l, k)}+N(l, k) e^{j \theta(l, k)}
\end{gathered}
$$

where $l$ and $k$ are the particular frame and frequency bin index with noisy, clean, and noise clean spectral amplitudes $R, X$, and $N$ and noisy, clean, and noise spectral phases $\vartheta, \alpha$, and $\theta$.

As opposed to using the traditional Rayleigh statistical models for both the speech prior and noise likelihood, the traditional Rayleigh speech prior given as

(3) $p(X, \alpha)=\frac{X}{\pi \sigma_{X}^{2}} \exp \left(-\frac{X^{2}}{\sigma_{X}^{2}}\right)$

is modified through the use of Chi speech priors (Johnson et al., 1994), where $\sigma_{X}^{2}$ is the speech spectral variance. Specifically, the Chi speech prior is given as

(4) $p(X, \alpha)=\frac{2}{\theta^{a} \Gamma(a)} X^{2 a-1} \exp \left(-\frac{X^{2}}{\theta}\right)$,

where $\sigma_{X}^{2}=\theta a$ with shape parameter $a$ and scaling parameter $\theta$ and $\Gamma(\bullet)$ is the gamma function.

With $a=0.5$ and $a=1,(4)$ is equivalent to the Half-Gaussian and Rayleigh distributions. The noise likelihood is still modeled as a Gaussian distribution given as

(5) $p(Y \mid X, \alpha)=\frac{1}{\pi \sigma_{N}^{2}} \exp \left(-\frac{\left|Y-X e^{j \alpha}\right|^{2}}{\sigma_{N}^{2}}\right)$, 
where $\sigma_{N}^{2}$ is the noise spectral variance. In order to simplify the notation, $\lambda=\sigma^{2}, \lambda_{X}=\sigma_{X}^{2}$, and $\lambda_{N}=\sigma_{N}^{2}$ is utilized as the spectral variances in the derivation of the WE with Chi speech prior estimator and WCOSH with Chi speech prior estimator.

\section{Perceptually-motivated cost functions with Chi speech priors}

\subsection{Weighted Euclidean (WE)}

From the work in Loizou (2005), the Weighted Euclidean (WE) cost function is given as

(6) $d_{\mathrm{WE}}(X, \hat{X})=(X-\hat{X})^{2} X^{p}$

with estimator equation

(7) $\hat{X}_{\mathrm{WE}}=\frac{\int_{0}^{\infty} \int_{0}^{2 \pi} X^{p+1} p(Y \mid X, \alpha) p(X, \alpha) d \alpha d X}{\int_{0}^{\infty} \int_{0}^{2 \pi} X^{p} p(Y \mid X, \alpha) p(X, \alpha) d \alpha d X}$,

where $p$ is the weighting law parameter. For $p=0,(7)$ is equivalent to the MMSE STSA estimator in Ephraim and Malah, 1984, Loizou, 2005, Gray et al., 1980.

Through the substitution of the statistical models in (4), (5) and using 8.431.5 and 8.406.1 in Gradshteyn and Ryzhik (2007), the spectral phase is integrated from the two integrals as

(8) $\int_{0}^{\infty} \int_{0}^{2 \pi} X^{p+1} p(Y \mid X, \alpha) p(X, \alpha) d \alpha d X \propto \int_{0}^{\infty} X^{p+2 a} \exp \left(-\frac{X^{2}}{\lambda_{a}}\right) J_{0}\left(2 i X \sqrt{\frac{v}{\lambda}}\right) d X$

and

(9) $\int_{0}^{\infty} \int_{0}^{2 \pi} X^{p} p(Y \mid X, \alpha) p(X, \alpha) d \alpha d X \propto \int_{0}^{\infty} X^{p+2 a-1} \exp \left(-\frac{X^{2}}{\lambda_{a}}\right) J_{0}\left(2 i X \sqrt{\frac{v}{\lambda}}\right) d X$,

where $\lambda$ is defined as

(10) $\frac{1}{\lambda}=\frac{1}{\lambda_{X}}+\frac{1}{\lambda_{N}}$

in (A.3) of Ephraim and Malah (1984), $J_{0}(\bullet)$ is the Oth-order Bessel function of the first-kind, and

(11) $\frac{1}{\lambda_{a}}=\frac{a}{\lambda_{X}}+\frac{1}{\lambda_{N}}$

which is equivalent to $1 / \lambda$ in (10) for $a=1$. By utilizing 6.631.1 and 9.212.1 in Gradshteyn and Ryzhik

(2007), (8), (9) are given as

$\int_{0}^{\infty} \int_{0}^{2 \pi} X^{p+1} p(Y \mid X, \alpha) p(X, \alpha) d \alpha d X \propto \frac{\Gamma\left(\frac{p+2 a+1}{2}\right)}{2} \lambda_{a}^{\frac{1}{2}(p+2 a+1)}{ }_{1} F_{1}\left(\frac{1-p-2 a}{2} ; 1 ;-\frac{v / \lambda}{1 / \lambda_{a}}\right)$

and

(13) $\int_{0}^{\infty} \int_{0}^{2 \pi} X^{p} p(Y \mid X, \alpha) p(X, \alpha) d \alpha d X \propto \frac{\Gamma\left(\frac{p+2 a}{2}\right)}{2} \lambda_{a}^{\frac{1}{2}(p+2 a)}{ }_{1} F_{1}\left(\frac{2-p-2 a}{2} ; 1 ;-\frac{v / \lambda}{1 / \lambda_{a}}\right)$

where ${ }_{1} F_{1}(\bullet ; \bullet ; \bullet)$ is the confluent hypergeometric function. With the combination of simplification of the integrals in (12), (13), the final form of the new WE estimator with Chi speech prior in (4) is given as 
(14) $\hat{X}_{\mathrm{WE}, \mathrm{CHI}}=G_{\mathrm{WE}, \mathrm{CHI}} R=\frac{\Gamma\left(\frac{p+2 a+1}{2}\right)}{\Gamma\left(\frac{p+2 a}{2}\right)} \frac{\sqrt{v_{a}}}{\gamma} \frac{1 F_{1}\left(\frac{1-p-2 a}{2} ; 1 ;-v \zeta_{a}\right)}{{ }_{1} F_{1}\left(\frac{2-p-2 a}{2} ; 1 ;-v \zeta_{a}\right)} R$,

where

(15) $v_{a}=\frac{\xi}{a+\xi} \gamma$

and

(16) $\zeta_{a}=\frac{1+\xi}{a+\xi}$

for $p+2 a>0$ with gain function $G_{\mathrm{WE}, \mathrm{CHI}}$ and a priori $\xi=\sigma_{X}^{2} / \sigma_{N}^{2}$ and a posteriori $\gamma=R^{2} / \sigma_{N}^{2}$ SNRs.

For $a=1$ and $p=0,(14)$ is exactly equivalent to the STSA estimator with Rayleigh speech prior (Ephraim and Malah, 1984).

\subsection{Weighted Cosh (WCOSH)}

In Loizou, 2005, the Weighted Cosh (WCOSH) cost function is given as

(17) $d_{\mathrm{WCOSH}}(X, \hat{X})=\left[\frac{X}{\hat{X}}+\frac{\hat{X}}{X}-1\right] X^{p}$

with estimator equation

(18) $\hat{X}_{\mathrm{WCOSH}}=\left[\frac{\int_{0}^{\infty} \int_{0}^{2 \pi} X^{p+1} p(Y \mid X, \alpha) p(X, \alpha) d \alpha d X}{\int_{0}^{\infty} \int_{0}^{2 \pi} X^{p-1} p(Y \mid X, \alpha) p(X, \alpha) d \alpha d X}\right]^{\frac{1}{2}}$,

where $p$ is the weighting law parameter. For $p=0,(18)$ is equivalent to the Cosh cost function given in Loizou, 2005, Gray et al., 1980. In order to determine the final estimator equation for the WCOSH with Chi speech prior, the integrals are derived in a same approach as with the WE with Chi speech prior estimator in (14).

By the substitution of the statistical models in (4), (5) and using 8.431.5 and 8.406.1 in Gradshteyn and Ryzhik (2007), the spectral phase is integrated from the two integrals as

(19) $\int_{0}^{\infty} \int_{0}^{2 \pi} X^{p+1} p(Y \mid X, \alpha) p(X, \alpha) d \alpha d X \propto \int_{0}^{\infty} X^{p+2 a} \exp \left(-\frac{X^{2}}{\lambda_{a}}\right) J_{0}\left(2 i X \sqrt{\frac{v}{\lambda}}\right) d X$

and

(20) $\int_{0}^{\infty} \int_{0}^{2 \pi} X^{p-1} p(Y \mid X, \alpha) p(X, \alpha) d \alpha d X \propto \int_{0}^{\infty} X^{p+2 a-2} \exp \left(-\frac{X^{2}}{\lambda_{a}}\right) J_{0}\left(2 i X \sqrt{\frac{v}{\lambda}}\right) d X$,

where $1 / \lambda_{a}$ is defined in (11). Through 6.631.1 and 9.212.1 in Gradshteyn and Ryzhik (2007), (19), (20) are given as

(21) $\int_{0}^{\infty} \int_{0}^{2 \pi} X^{p+1} p(Y \mid X, \alpha) p(X, \alpha) d \alpha d X \propto \frac{\Gamma\left(\frac{p+2 a+1}{2}\right)}{2} \lambda_{a}^{\frac{1}{2}(p+2 a+1)}{ }_{1} F_{1}\left(\frac{1-p-2 a}{2} ; 1 ;-\frac{v / \lambda}{1 / \lambda_{a}}\right)$

and

(22) $\int_{0}^{\infty} \int_{0}^{2 \pi} X^{p-1} p(Y \mid X, \alpha) p(X, \alpha) d \alpha d X \propto \frac{\Gamma\left(\frac{p+2 a-1}{2}\right)}{2} \lambda_{a}^{\frac{1}{2}(p+2 a-1)}{ }_{1} F_{1}\left(\frac{3-p-2 a}{2} ; 1 ;-\frac{v / \lambda}{1 / \lambda_{a}}\right)$.

With the combination of simplification of the integrals in (21), (22) and using $v_{a}$ and $\zeta_{a}$ in (15) and equation, the final form of the new WCOSH estimator with Chi speech prior in (4) is given as 
(23) $\hat{X}_{\mathrm{WCOSH}, \mathrm{CHI}}=G_{\mathrm{WCOSH}, \mathrm{CHI}} R=\left[\frac{\Gamma\left(\frac{p+2 a+1}{2}\right)}{\Gamma\left(\frac{p+2 a-1}{2}\right)}\right]^{\frac{1}{2}} \frac{\sqrt{v_{a}}}{\gamma}\left[\frac{{ }_{1} F_{1}\left(\frac{1-p-2 a}{2} ; 1 ;-v \zeta_{a}\right)}{{ }_{1} F_{1}\left(\frac{3-p-2 a}{2} ; 1 ;-v \zeta_{a}\right)}\right]^{\frac{1}{2}} R$

for $p+2 a>1$ with gain function $G_{\mathrm{WCOSH}, \mathrm{CHI}}$. For $a=1$ and $p=0,(23)$ is similar to the LSA estimator with Rayleigh speech prior (Ephraim and Malah, 1985). By comparing the WE and WCOSH estimators given in (18), (19), (20), (21), (22), (23), the only differences consist of the integral in the denominator and square root.

Fig. 1, Fig. 2, Fig. 3(WE with Chi speech prior estimator) and Fig. 4, Fig. 5, Fig. 6 (WCOSH with Chi speech prior estimator) present the gain functions $G_{\mathrm{WE}, \mathrm{CHI}}$ and $G_{\mathrm{WCOSH}, \mathrm{CHI}}$ for the WE and WCOSH with Chi speech prior estimators given in (14), (23) using representative weighting law parameters of $p_{\mathrm{WE}}=\{-1.00,-0.50,-0.25\}$ and $p_{\mathrm{WCOSH}}=\{-0.75,-0.50,-0.25\}$ as a function of instantaneous SNR $\gamma_{k}-1$ for three fixed $a$ priori SNR $\xi_{k}$ values of 0,5 , and $10 \mathrm{~dB}$ and valid shaping parameter $a$ values.

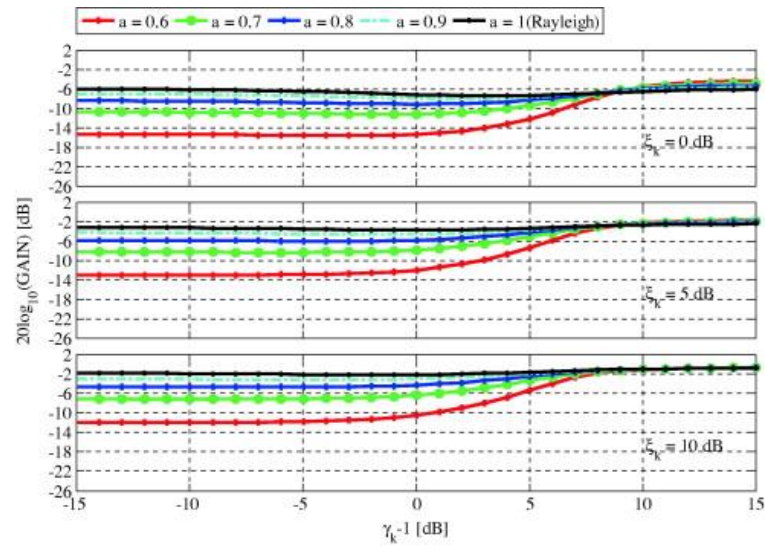

Fig. 1. Gain curves for WE $(p=-1.0)$ estimator with Chi prior.

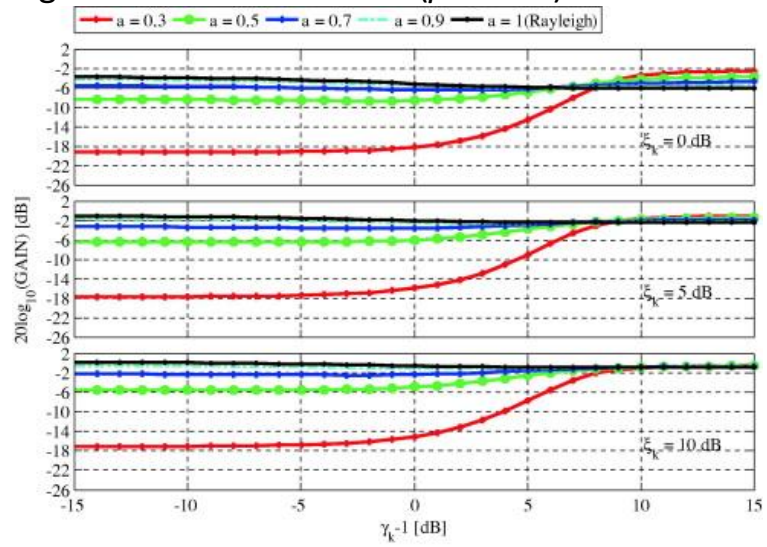

Fig. 2. Gain curves for WE $(p=-0.50)$ estimator with Chi prior.

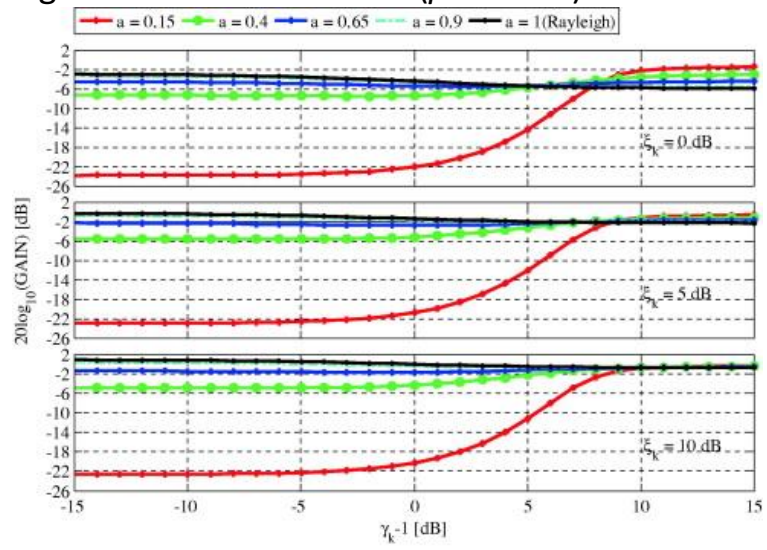


Fig. 3. Gain curves for WE ( $p=-0.25)$ estimator with Chi prior.

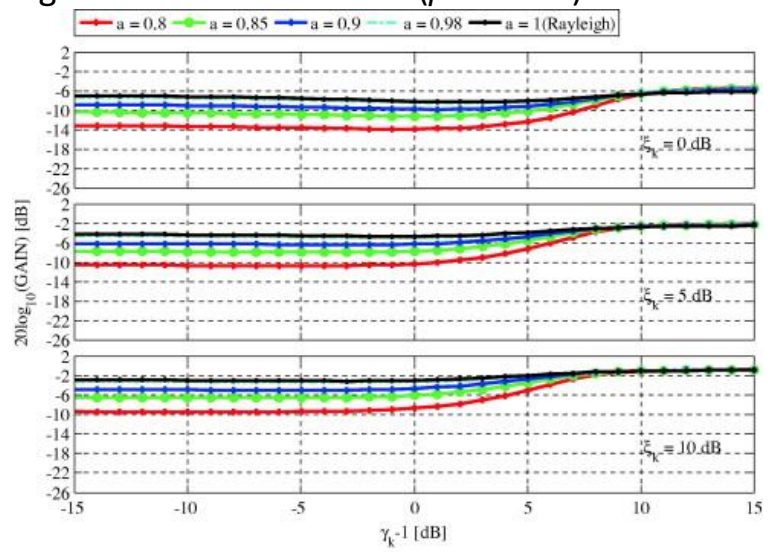

Fig. 4. Gain curves for WCOSH $(p=-0.75)$ estimator with Chi prior.

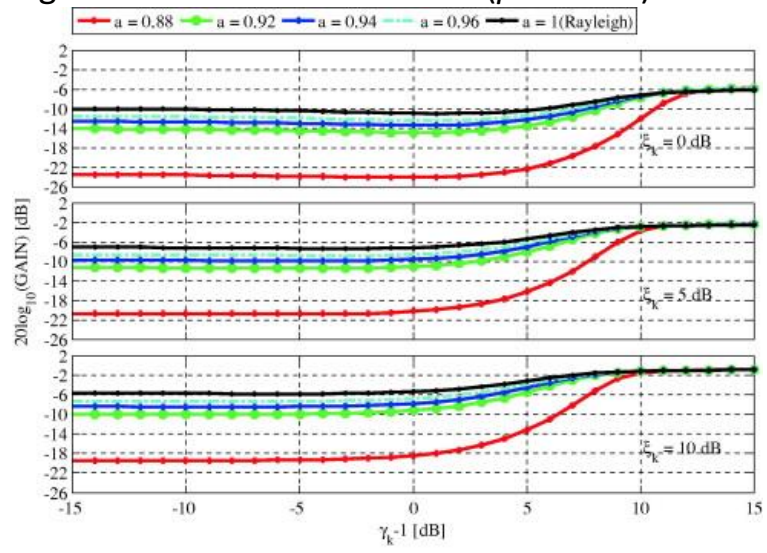

Fig. 5. Gain curves for WCOSH $(p=-0.50)$ estimator with Chi prior.

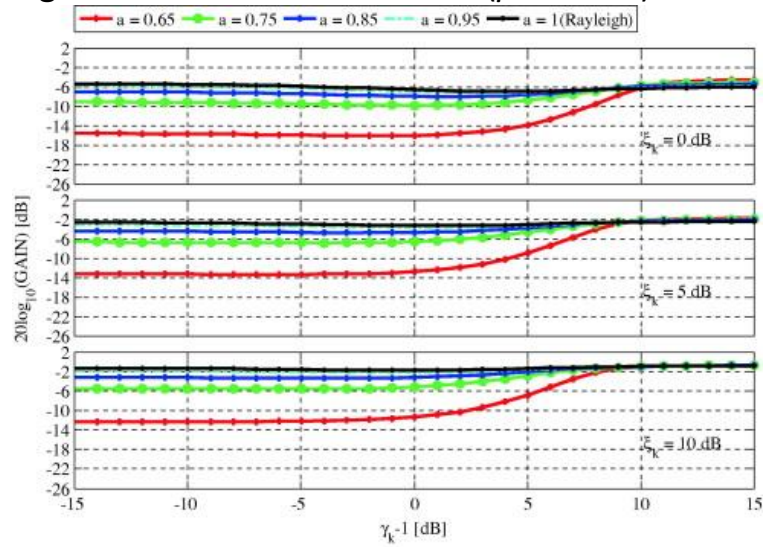

Fig. 6. Gain curves for WCOSH $(p=-0.25)$ estimator with Chi prior.

From the gain curves, there are several interesting observations to note from both the WE and WCOSH with Chi and Rayleigh speech prior estimators. Based on both sets of estimators across the different $a$ priori SNR $\xi_{k}$, the gains were smaller in value (more attenuation) as the shaping parameter $a$ approached its limiting value with a decrease in the instantaneous a posteriori SNR $\gamma_{k}-1$. As the shaping parameter $a \rightarrow 1$, which is the Rayleigh speech prior, the gains had a flatter shape and larger value (less attenuation). Regardless of the $a$ priori SNR $\xi_{k}$ and shaping parameter $a$, the gains all eventually converged to approximately $0--6 \mathrm{~dB}$ at around an a posteriori SNR of 8-10 dB with an increase of the instantaneous a posteriori SNR $\gamma_{k}-1$, which was essentially independent of the weighting law parameter $p$. With the WE with Chi speech prior estimator, the increase in the weighting law parameter $p$, which in turn causes an increase in the range of valid shaping 
parameters $a$, generated gains with more attenuation at lower instantaneous a posteriori SNR $\gamma_{k}-1$ (and less attenuation at higher instantaneous a posteriori SNR $\gamma_{k}-1$ ) using the limiting value of the shaping parameter $a$. The gains with an increase of weighting law parameter $p$ and shaping parameter $a \rightarrow 1$ (Rayleigh speech prior) had less attenuation at lower instantaneous a posteriori SNR $\gamma_{k}-1$ and no substantial change in attenuation at higher instantaneous a posteriori SNR $\gamma_{k}-1$. For the WCOSH with Chi speech prior estimator, the gains were much more dependent on the a priori SNR $\xi_{k}$ than the WE with Chi speech prior estimator. For a particular weighting law parameter $p$ across all shaping parameter $a$, the gains had less attenuation with an increase in the a priori SNR $\xi_{k}$. By comparing the same weighting law parameter $p=-0.50$ (Fig. 2, Fig. 5) and $p=-0.25$ (Fig. 3, Fig. 6) across the WE and WCOSH with Chi speech prior estimators, the gains associated with the WE with Chi speech prior estimator had significantly more attenuation at lower instantaneous a posteriori SNR $\gamma_{k}-1$ (and similar attenuation at higher lower instantaneous $a$ posteriori SNR $\gamma_{k}-1$ ) than the gains associated with the WCOSH with Chi speech prior estimator.

\section{Experiments and results}

The proposed WE and WCOSH with Chi speech prior optimal estimators given in (14), (23) were evaluated using the objective measures of Segmental Signal-to-Noise Ratio (SSNR) Papamichalis, 1987, Perceptual Evaluation of Speech Quality (PESQ) ITU, 2003, Hu and Loizou, 2007, Hu and Loizou, 2008, Rix et al., 2001, and Signal-to-Noise Ratio (SNR) Loss Ma and Loizou, 2011 to access noise reduction, overall speech quality, and speech intelligibility, where PESQ and SNR Loss have a range of 0.5-4.5 (higher scores indicate better performance) and 0.0-2.0 (lower scores indicate better performance). In particular, the performance is given via SSNR, PESQ, and SNR LosS improvements, where the improvements are calculated as SSNR/PESQ/SNR Loss output (enhanced signal) minus SSNR/PESQ/SNR Loss input (noisy signal). Clean and noisy speech were taken from the noisy speech corpus (NOIZEUS) Hu and Loizou, 2007, which contains 30 IEEE sentences (Subcommittee, 1969) (produced by three male and three female speakers) corrupted by eight different real-world noises at different SNRs ranging from 0 to $15 \mathrm{~dB}$ at increments of $5 \mathrm{~dB}$, where the noises were taken from the AURORA database (Pearce and Hirsch, 2000), which includes airport, babble, car, exhibition, restaurant, station, street, and train noises. The analysis conditions consisted of frames of 256 samples $(25.6 \mathrm{~ms}$ ) with $50 \%$ overlap using Hanning windows. Noise estimation was performed on an initial silence of 5 frames. The decision-directed (DD) Ephraim and Malah, 1984 smoothing approach was utilized to estimate $\xi$ with $\alpha_{\mathrm{SNR}}=0.98$ using thresholds of $\xi_{\min }=10^{-25 / 10}$ and $\gamma_{\min }=40$. In order to evaluate the performance, the enhanced signals were reconstructed using the overlap-add technique. The shape parameter $a$ in the Chi speech prior was varied for specific weighting law parameters $p$ to determine its effect on enhancement, quality, and intelligibility with results averaged over 30 utterances across the 8 different noises at input SNRs of 0,5 , and $10 \mathrm{~dB}$. As recommended by Loizou (2005), $p_{\mathrm{WE}}=-1$ and $p_{\mathrm{WCOSH}}=-0.5$ were selected as the weighting law parameter $p$ to achieve the best overall speech quality in the enhancement process.

Fig. 7, Fig. 8 illustrate the SSNR improvements for the WE and WCOSH with Chi speech prior estimators at various input SNRs, noises, and shaping parameter $a$ with particular weighting law parameter $p$. The WE and WCOSH with Chi speech prior estimators consistently produced 2-3 dB (0 dB input SNR), 1-2 dB (5 dB input SNR), and 0-2 dB (10 dB input SNR) over the baseline WE and WCOSH with Rayleigh speech prior estimators, which typically occurred at the limiting value of the shaping parameter $a$ for the corresponding weighting law parameter $p$ of $a \rightarrow 0.50\left(p_{\mathrm{WE}}=-1.0\right)$ and $a \rightarrow 0.75\left(p_{\mathrm{WCOSH}}=-0.5\right)$. At the limiting shaping parameter $a$, the WE and WCOSH with Chi speech prior estimators achieved maximum SSNR improvements of 9-13 dB (0 dB input SNR), 6-9 dB (5 dB input SNR), and 4-5 dB (10 dB input SNR) across the car, train, station, exhibition, street, babble, and airport noises. In comparing the WE and WCOSH with Chi speech prior estimators, the WE with Chi speech prior estimator had slightly better SSNR improvement performance over the WCOSH with Chi speech prior estimator for noise reduction. 


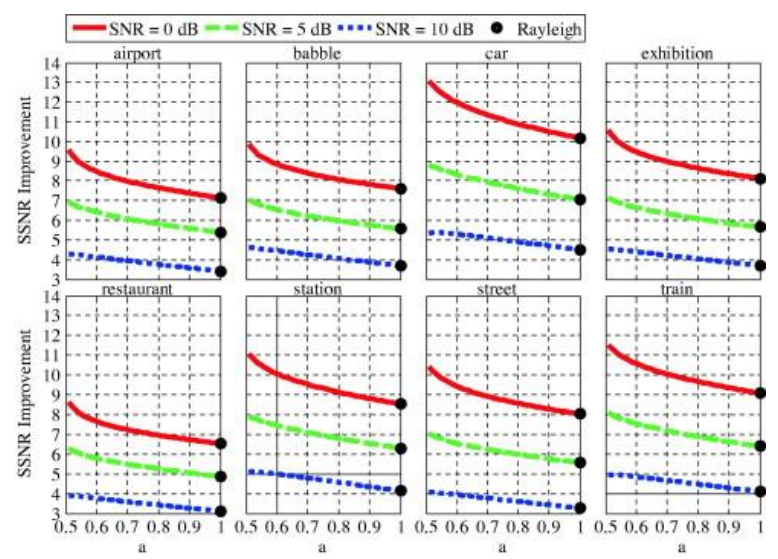

Fig. 7. SSNR improvements for MMSE WE estimator with Chi prior $(p=-1)$.

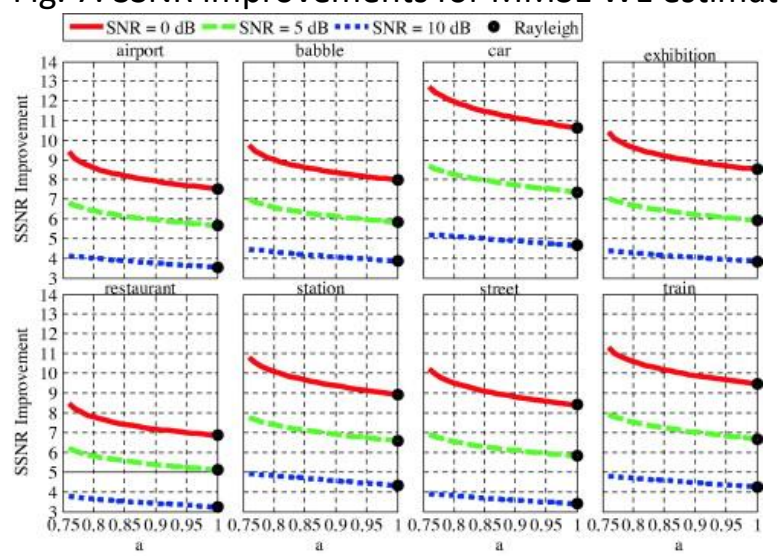

Fig. 8. SSNR improvements for MMSE WCOSH estimator with Chi prior $(p=-0.50)$.

Fig. 9, Fig. 10 present the PESQ improvements for the WE and WCOSH with Chi speech prior estimators at various input SNRs, noises, and shaping parameter $a$ with particular weighting law parameter $p$. In a similar fashion to the SSNR improvements, the WE and WCOSH with Chi speech prior estimators generated 0.00-0.03 and 0.00-0.01 gains over the baseline WE and WCOSH estimators with Rayleigh speech prior with the most pronounced improvements occurring at input SNRs of 5 and $10 \mathrm{~dB}$. In contrast to the SSNR improvements that were almost exclusively dependent on the limiting shaping parameter $a$, the PESQ improvements diminished at $a=0.70-0.80$ (WE with Chi speech prior estimator) and $a=0.85-0.90$ (WCOSH with Chi speech prior estimator). For both the WE and WCOSH with Chi prior estimators, the maximum PESQ improvements ranged from 0.20-0.55 (5 dB input SNR), 0.20-0.50 (10 dB input SNR), and 0.14-0.48 (0 dB input SNR) across the restaurant, airport, babble, street, exhibition, station, train, and car noises. After examination of the WE and WCOSH with Chi speech prior estimators, the WE with Chi speech prior estimator had slightly better PESQ improvement performance over the WCOSH with Chi speech prior estimator for speech quality. 


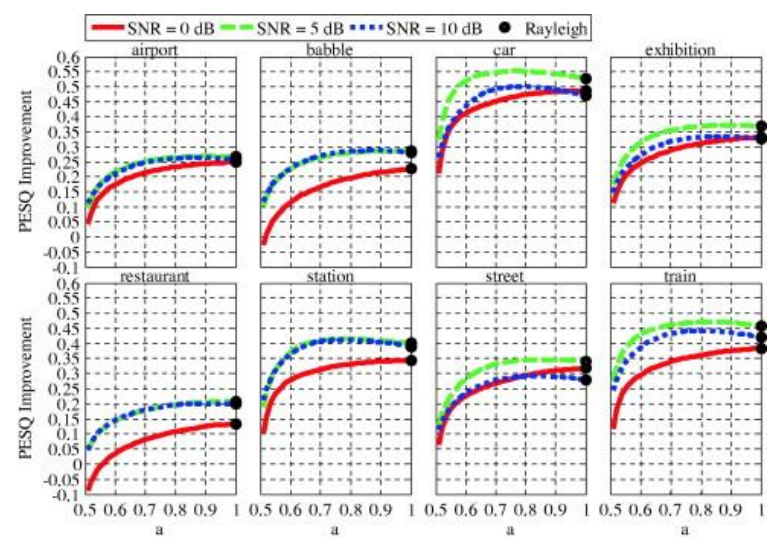

Fig. 9. PESQ improvements for MMSE WE estimator with Chi prior $(p=-1)$.

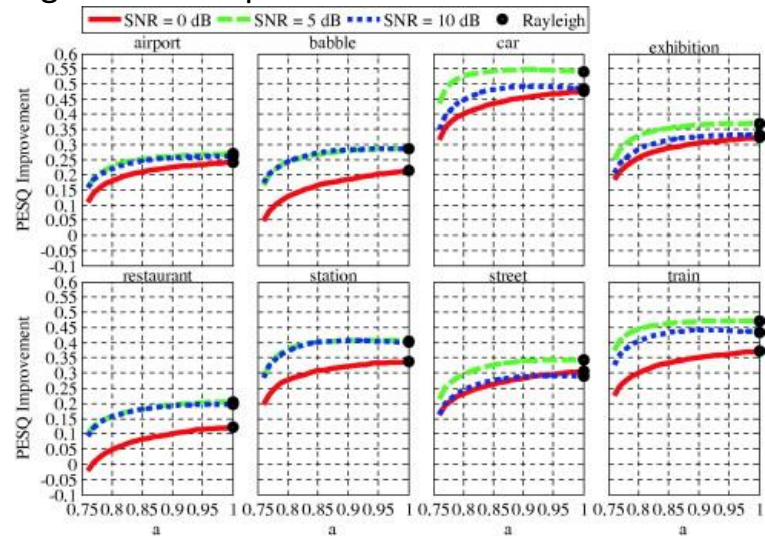

Fig. 10. PESQ improvements for MMSE WCOSH estimator with Chi prior $(p=-0.50)$.

Fig. 11, Fig. 12 demonstrate the SNR Loss improvements for the WE and WCOSH with Chi speech prior estimators at various input SNRs, noises, and shaping parameter $a$ with particular weighting law parameter $p$. The WE and WCOSH with Chi speech prior estimators typically yielded 0-0.01 (0 dB input SNR), 0-0.005 (5 dB input SNR), and 0-0.005 (10 dB input SNR) over the corresponding baseline WE and WCOSH with Rayleigh speech prior estimators, which occurred at a wide range of shaping parameters $a$. In contrast to the SSNR and PESQ improvements, the SNR Loss improvements were most noticeable at input SNRs of 5, 0, and $10 \mathrm{~dB}$ and car, station, babble, airport, exhibition, restaurant, train, and street noises. In more specific terms, the WE and WCOSH with Chi speech prior estimators realized maximum SNR Loss improvements of $-0.1150-0.0950(0 \mathrm{~dB}$ input SNR), -0.1000--0.0905 (5 dB input SNR), and -0.0905--0.0800. From the WE and WCOSH with Chi speech prior estimators, the WE with Chi speech prior estimator often had larger decreases in SNR Loss over the baseline Rayleigh speech prior estimators than the WCOSH with Chi speech prior estimator.

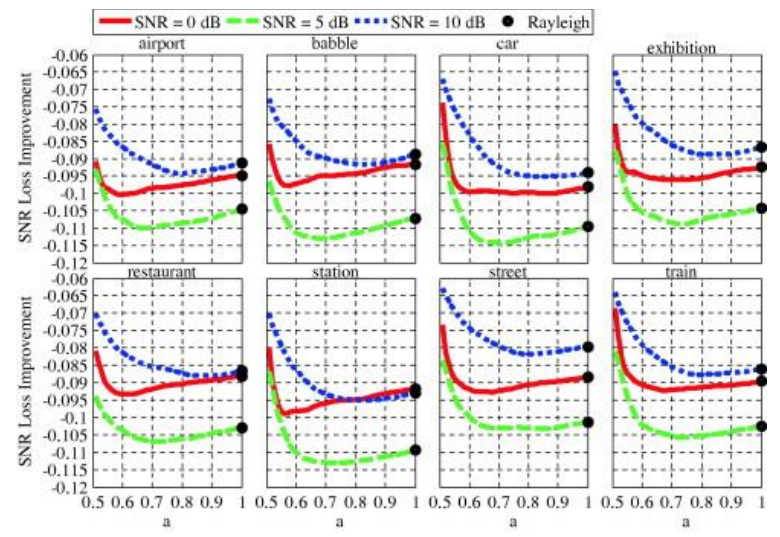


Fig. 11. SNR Loss improvements for MMSE WE estimator with Chi prior $(p=-1)$.

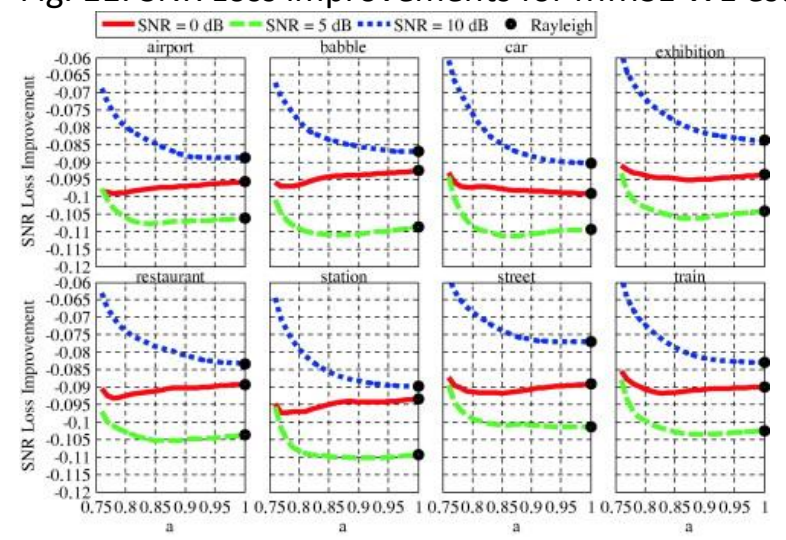

Fig. 12. SNR Loss improvements for MMSE WCOSH estimator with Chi prior $(p=-0.50)$.

Table 1, Table 2, Table 3, Table 4, Table 5, Table 6 show the SSNR improvement, PESQ improvement, and SNR Loss improvement for the WE and WCOSH with Chi speech prior estimators for two additional and representative weighting law parameters $p$. Whereas the WE with Chi speech prior estimator was examined with the weighting law parameters $p=-0.50(a>0.25)$ and $p=-0.25(a>0.125)$, the WCOSH with Chi speech prior estimator was examined with the weighting law parameters $p=-0.75(a>0.875)$ and $p=-0.25(a>0.625)$ according to the relationships $p+2 a>0$ and $p+2 a>1$. For each weighting law parameter $p$ of the WCOSH and WE with Chi speech prior estimators at the particular noise and input SNR, the SSNR improvement, PESQ improvement, and SNR Loss improvement results are provided alongside their corresponding shaping parameter $a$, where the shaping parameter $a \rightarrow 1$ represents the baseline WE and WCOSH with Rayleigh speech prior estimators. In terms of SSNR improvements, the WE and WCOSH with Chi speech prior estimators generally produced $0.5-2.5 \mathrm{~dB}$ gains over the baseline WE and WCOSH with Rayleigh speech prior estimators. As the weighting law parameter $p$ was decreased in value, the SSNR improvement increased in value, where the maximum SSNR improvement ranged from 6 to $9 \mathrm{~dB}$ across the car, train, and babble noises. The WCOSH with Chi speech prior typically had less performance gains over the baseline WCOSH with Rayleigh speech prior because of the higher baseline SSNR improvements. For each the WE and WCOSH with Chi speech prior estimators, the limiting factor in SSNR improvement was the lower bound of the shaping parameters $a$. For the PESQ improvements, the WE and WCOSH with Chi speech prior estimators generated upwards of 0.14 gains over the baseline WE and WCOSH with Rayleigh speech prior estimators. In a similar way to the SSNR improvements, the increase in the weighting law parameter $p$ caused a decrease in PESQ improvement. The maximum PESQ improvement ranged from 0.24 to 0.56 across the car, train, and babble noises, where the shaping parameter $a$ reached the maximum at $a=0.50-0.70$ (WE with Chi speech prior estimator) and $a=0.90-0.99$ (WCOSH with Chi speech prior estimator). In general, the WCOSH with Chi speech prior estimator did not always follow the same relationship between the weighting law parameter $p$ and PESQ improvement as the WE with Chi speech prior estimator. With the SNR Loss improvements, the WE and WCOSH with Chi speech prior estimators supplied nearly 0.019 gains over the baseline WE and WCOSH with Rayleigh speech prior estimators. As with SSNR improvement and PESQ improvement, the SNR LosS improvement decreased in value with an increase in the weighting law parameter $p$ value. The car, babble, and train noises achieved maximum SNR Loss improvements of $-0.088-0.110$, which occurred in the range of $a=0.25-0.45$ (WE with Chi speech prior estimator) and $a / 1.00$ (WCOSH with Chi speech prior estimator). In most cases, the WCOSH with Chi speech prior estimator did not produce nearly as pronounced SNR LosS improvement gains compared to the WE with Chi speech prior estimator over the baseline and WE and WCOSH with Rayleigh speech prior estimators. 
Table 1. SSNR improvements for MMSE WE estimator with Chi prior $(p=-0.50$ and $p=-0.25)$.

\begin{tabular}{|c|c|c|c|c|c|c|c|c|c|c|c|c|}
\hline SNR [dB] & Babble & & & & Car & & & & Train & & & \\
\hline & $p=-0.50$ & & $p=-0.25$ & & $p=-0.50$ & & $p=-0.25$ & & $p=-0.50$ & & $p=-0.25$ & \\
\hline \multirow[t]{2}{*}{0} & 6.29 & 1.00 & 5.55 & 1.00 & 8.51 & 1.00 & 7.52 & 1.00 & 7.64 & 1.00 & 6.77 & 1.00 \\
\hline & 8.92 & 0.25 & 7.85 & 0.13 & 12.33 & 0.25 & 11.27 & 0.13 & 10.82 & 0.25 & 9.97 & 0.13 \\
\hline \multirow[t]{2}{*}{5} & 4.54 & 1.00 & 3.97 & 1.00 & 5.85 & 1.00 & 5.13 & 1.00 & 5.23 & 1.00 & 4.60 & 1.00 \\
\hline & 6.55 & 0.25 & 5.92 & 0.13 & 8.44 & 0.25 & 7.87 & 0.13 & 7.62 & 0.25 & 7.02 & 0.13 \\
\hline \multirow[t]{2}{*}{10} & 2.98 & 1.00 & 2.56 & 1.00 & 3.74 & 1.00 & 3.26 & 1.00 & 3.40 & 1.00 & 2.96 & 1.00 \\
\hline & 4.53 & 0.25 & 4.27 & 0.13 & 5.40 & 0.26 & 5.27 & 0.13 & 4.93 & 0.25 & 4.74 & 0.13 \\
\hline \multirow[t]{2}{*}{ AVG. } & 4.60 & 1.00 & 4.03 & 1.00 & 6.03 & 1.00 & 5.30 & 1.00 & 5.42 & 1.00 & 4.78 & 1.00 \\
\hline & 6.67 & 0.25 & 6.01 & 0.13 & 8.72 & 0.25 & 8.14 & 0.13 & 7.79 & 0.25 & 7.24 & 0.13 \\
\hline
\end{tabular}

Table 2. SSNR improvements for MMSE WCOSH estimator with Chi prior ( $p=-0.75$ and $p=-0.25$ ).

\begin{tabular}{|l|l|l|l|l|l|l|l|l|l|l|l|l|}
\hline SNR [dB] & Babble & & & & Car & & & \multicolumn{3}{l|}{ Train } & & \\
\hline & $\boldsymbol{p}=-\mathbf{0 . 7 5}$ & & $\boldsymbol{p}=-\mathbf{0 . 2 5}$ & & $\boldsymbol{p}=-\mathbf{0 . 7 5}$ & & $\boldsymbol{p}=-\mathbf{0 . 2 5}$ & & $\boldsymbol{p}=\mathbf{- 0 . 7 5}$ & & $\boldsymbol{p}=\mathbf{- 0 . 2 5}$ & \\
\hline 0 & 8.77 & 1.00 & 7.27 & 1.00 & 11.54 & 1.00 & 9.77 & 1.00 & 10.25 & 1.00 & 8.72 & 1.00 \\
\hline & 9.83 & 0.88 & 9.28 & 0.63 & 12.73 & 0.88 & 12.37 & 0.63 & 11.31 & 0.88 & 10.93 & 0.63 \\
\hline 5 & 6.38 & 1.00 & 5.30 & 1.00 & 7.98 & 1.00 & 6.75 & 1.00 & 7.27 & 1.00 & 6.11 & 1.00 \\
\hline & 7.05 & 0.88 & 6.76 & 0.63 & 8.67 & 0.88 & 8.50 & 0.63 & 7.95 & 0.88 & 7.74 & 0.63 \\
\hline 10 & 4.16 & 1.00 & 3.50 & 1.00 & 4.93 & 1.00 & 4.30 & 1.00 & 4.53 & 1.00 & 3.91 & 1.00 \\
\hline & 4.43 & 0.88 & 4.43 & 0.63 & 5.11 & 0.88 & 5.21 & 0.63 & 4.76 & 0.88 & 4.80 & 0.63 \\
\hline AVG. & 6.44 & 1.00 & 5.36 & 1.00 & 8.15 & 1.00 & 6.94 & 1.00 & 7.35 & 1.00 & 6.25 & 1.00 \\
\hline & 7.10 & 0.88 & 6.82 & 0.63 & 8.84 & 0.88 & 8.69 & 0.63 & 8.01 & 0.88 & 7.82 & 0.63 \\
\hline
\end{tabular}

Table 3. PESQ improvements for MMSE WE estimator with Chi prior ( $p=-0.50$ and $p=-0.25)$.

\begin{tabular}{|l|l|l|l|l|l|l|l|l|l|l|l|l|}
\hline SNR [dB] & Babble & & & & Car & & & & Train & & \\
\hline & $\boldsymbol{p}=-\mathbf{0 . 5 0}$ & & $\boldsymbol{p}=-\mathbf{0 . 2 5}$ & & $\boldsymbol{p}=-\mathbf{0 . 5 0}$ & & $\boldsymbol{p}=\mathbf{- 0 . 2 5}$ & & $\boldsymbol{p}=\mathbf{- 0 . 5 0}$ & & $\boldsymbol{p}=\mathbf{- 0 . 2 5}$ & \\
\hline 0 & 0.23 & 1.00 & 0.22 & 1.00 & 0.47 & 1.00 & 0.42 & 1.00 & 0.38 & 1.00 & 0.35 & 1.00 \\
\hline & 0.23 & 0.86 & 0.23 & 0.66 & 0.50 & 0.61 & 0.51 & 0.39 & 0.39 & 0.68 & 0.39 & 0.51 \\
\hline 5 & 0.26 & 1.00 & 0.23 & 1.00 & 0.47 & 1.00 & 0.41 & 1.00 & 0.39 & 1.00 & 0.34 & 1.00 \\
\hline & 0.28 & 0.65 & 0.27 & 0.47 & 0.56 & 0.44 & 0.55 & 0.29 & 0.46 & 0.50 & 0.45 & 0.33 \\
\hline 10 & 0.22 & 1.00 & 0.19 & 1.00 & 0.39 & 1.00 & 0.33 & 1.00 & 0.35 & 1.00 & 0.30 & 1.00 \\
\hline & 0.29 & 0.54 & 0.28 & 0.38 & 0.52 & 0.48 & 0.53 & 0.31 & 0.44 & 0.50 & 0.44 & 0.33 \\
\hline AVG. & 0.24 & 1.00 & 0.21 & 1.00 & 0.44 & 1.00 & 0.39 & 1.00 & 0.37 & 1.00 & 0.33 & 1.00 \\
\hline
\end{tabular}


Table 4. PESQ improvements for MMSE WCOSH estimator with Chi prior ( $p=-0.75$ and $p=-0.25$ ).

\begin{tabular}{|l|l|l|l|l|l|l|l|l|l|l|l|l|}
\hline SNR [dB] & Babble & & & & Car & & & & Train & & \\
\hline & $\boldsymbol{p}=-\mathbf{0 . 7 5}$ & & $\boldsymbol{p}=-\mathbf{0 . 2 5}$ & & $\boldsymbol{p}=-\mathbf{0 . 7 5}$ & & $\boldsymbol{p}=-\mathbf{0 . 2 5}$ & & $\boldsymbol{p}=\mathbf{- 0 . 7 5}$ & & $\boldsymbol{p}=\mathbf{- 0 . 2 5}$ & \\
\hline 0 & 0.17 & 1.00 & 0.23 & 1.00 & 0.43 & 1.00 & 0.49 & 1.00 & 0.33 & 1.00 & 0.39 & 1.00 \\
\hline & 0.17 & 0.99 & 0.23 & 0.99 & 0.43 & 0.99 & 0.49 & 0.89 & 0.33 & 0.99 & 0.39 & 0.95 \\
\hline 5 & 0.27 & 1.00 & 0.28 & 1.00 & 0.54 & 1.00 & 0.52 & 1.00 & 0.47 & 1.00 & 0.44 & 1.00 \\
\hline & 0.27 & 0.99 & 0.28 & 0.87 & 0.54 & 0.99 & 0.55 & 0.75 & 0.47 & 0.99 & 0.47 & 0.78 \\
\hline 10 & 0.27 & 1.00 & 0.27 & 1.00 & 0.48 & 1.00 & 0.46 & 1.00 & 0.43 & 1.00 & 0.41 & 1.00 \\
\hline & 0.27 & 0.99 & 0.29 & 0.82 & 0.48 & 0.99 & 0.50 & 0.77 & 0.43 & 0.99 & 0.44 & 0.76 \\
\hline AVG. & 0.24 & 1.00 & 0.26 & 1.00 & 0.48 & 1.00 & 0.49 & 1.00 & 0.41 & 1.00 & 0.41 & 1.00 \\
\hline & 0.24 & 0.99 & 0.27 & 0.89 & 0.48 & 0.99 & 0.51 & 0.80 & 0.41 & 0.99 & 0.43 & 0.83 \\
\hline
\end{tabular}

Table 5. SNR Loss improvements for MMSE WE estimator with Chi prior ( $p=-0.50$ and $p=-0.25)$.

\begin{tabular}{|l|l|l|l|l|l|l|l|l|l|l|l|l|}
\hline SNR [dB] & Babble & & & & Car & & & & Train & & \\
\hline & $\boldsymbol{p}=-\mathbf{0 . 5 0}$ & & $\boldsymbol{p}=-\mathbf{0 . 2 5}$ & & $\boldsymbol{p}=-\mathbf{0 . 5 0}$ & & $\boldsymbol{p}=-\mathbf{0 . 2 5}$ & & $\boldsymbol{p}=-\mathbf{0 . 5 0}$ & & $\boldsymbol{p}=-\mathbf{0 . 2 5}$ & \\
\hline 0 & -0.086 & 1.00 & -0.081 & 1.00 & -0.093 & 1.00 & -0.086 & 1.00 & -0.084 & 1.00 & -0.079 & 1.00 \\
\hline & -0.094 & 0.28 & -0.091 & 0.15 & -0.101 & 0.50 & -0.100 & 0.31 & -0.092 & 0.42 & -0.089 & 0.30 \\
\hline 5 & -0.101 & 1.00 & -0.095 & 1.00 & -0.104 & 1.00 & -0.098 & 1.00 & -0.096 & 1.00 & -0.090 & 1.00 \\
\hline & -0.112 & 0.36 & -0.109 & 0.33 & -0.118 & 0.40 & -0.116 & 0.31 & -0.108 & 0.44 & -0.105 & 0.31 \\
\hline 10 & -0.083 & 1.00 & -0.078 & 1.00 & -0.092 & 1.00 & -0.088 & 1.00 & -0.084 & 1.00 & -0.080 & 1.00 \\
\hline & -0.101 & 0.50 & -0.106 & 0.33 & -0.108 & 0.48 & -0.115 & 0.35 & -0.098 & 0.49 & -0.104 & 0.33 \\
\hline AVG. & -0.090 & 1.00 & -0.085 & 1.00 & -0.096 & 1.00 & -0.091 & 1.00 & -0.088 & 1.00 & -0.083 & 1.00 \\
\hline & -0.102 & 0.38 & -0.088 & 0.27 & -0.109 & 0.46 & -0.110 & 0.32 & -0.099 & 0.45 & -0.099 & 0.31 \\
\hline
\end{tabular}

Table 6. SNR Loss improvements for MMSE WCOSH estimator with Chi prior ( $p=-0.75$ and $p=-0.25$ ).

\begin{tabular}{|l|l|l|l|l|l|l|l|l|l|l|l|l|}
\hline SNR [dB] & Babble & & & & Car & & & & Train & & \\
\hline & $\boldsymbol{p}=-\mathbf{0 . 7 5}$ & & $\boldsymbol{p}=-\mathbf{0 . 2 5}$ & & $\boldsymbol{p}=-\mathbf{0 . 7 5}$ & & $\boldsymbol{p}=-\mathbf{0 . 2 5}$ & & $\boldsymbol{p}=-\mathbf{0 . 7 5}$ & & $\boldsymbol{p}=\mathbf{- 0 . 2 5}$ & \\
\hline 0 & -0.094 & 1.00 & -0.091 & 1.00 & -0.096 & 1.00 & -0.097 & 1.00 & -0.091 & 1.00 & -0.088 & 1.00 \\
\hline & -0.097 & 0.90 & -0.097 & 0.63 & -0.096 & 0.99 & -0.100 & 0.80 & -0.091 & 0.97 & -0.092 & 0.68 \\
\hline 5 & -0.110 & 1.00 & -0.106 & 1.00 & -0.109 & 1.00 & -0.108 & 1.00 & -0.102 & 1.00 & -0.101 & 1.00 \\
\hline & -0.110 & 0.99 & -0.112 & 0.71 & -0.109 & 0.99 & -0.113 & 0.72 & -0.102 & 0.99 & -0.105 & 0.73 \\
\hline 10 & -0.081 & 1.00 & -0.086 & 1.00 & -0.082 & 1.00 & -0.093 & 1.00 & -0.076 & 1.00 & -0.085 & 1.00 \\
\hline
\end{tabular}




\begin{tabular}{|l|l|l|l|l|l|l|l|l|l|l|l|l|}
\hline & -0.081 & 0.99 & -0.090 & 0.82 & -0.082 & 0.99 & -0.095 & 0.87 & -0.076 & 0.99 & -0.087 & 0.86 \\
\hline AVG. & -0.095 & 1.00 & -0.094 & 1.00 & -0.096 & 1.00 & -0.099 & 1.00 & -0.090 & 1.00 & -0.091 & 1.00 \\
\hline & -0.096 & 0.96 & -0.100 & 0.72 & -0.096 & 0.99 & -0.103 & 0.80 & -0.090 & 0.98 & -0.095 & 0.76 \\
\hline
\end{tabular}




\section{Conclusion}

In this paper, the authors derived novel perceptually-motivated WE and WCOSH estimators using more appropriate Chi speech prior as a substitute for the traditional Rayleigh speech prior to model the speech spectral amplitude. Fundamentally, the goal of the work is to capitalize on the mutual benefits of the WE and WCOSH cost functions and Chi distributions for the speech prior to provide gains in all phases of enhancement. The WE and WCOSH with Chi speech prior estimators incorporated weighting law and shape parameters on the cost functions and distributions. Instead of measuring the performance simply with the SSNR objective quality metric to determine the amount of noise reduction, the estimators were evaluated using the PESQ and SNR Loss objective quality metrics to ascertain the level of overall speech quality and speech intelligibility compared to the original noisy signals corrupted by input SNRs of 0,5 , and $10 \mathrm{~dB}$ across airport, babble, car, exhibition, restaurant, station, street, and train noises. With the WE and WCOSH with standard Rayleigh speech prior estimators serving as the baseline results, the experimental results indicated that the new WE and WCOSH with Chi speech prior estimators provided significant gains in noise reduction and noticeable gains in overall speech quality and speech intelligibility improvements. Generally, the best results for the various objective quality metrics occurred for a particular weighting law parameter at the limiting value of the shaping parameter at lower input SNRs (SSNR improvement) and various values of the shaping parameter at higher input SNRs (PESQ improvement and SNR Loss improvement). In more specific terms, the WE and WCOSH with Chi speech prior estimators consistently produced upwards of approximately $3 \mathrm{~dB}$ (SSNR improvement), 0.03 (PESQ improvement), and 0.005 (SNR Loss improvement) over the baseline WE and WCOSH with Rayleigh speech prior estimators. In comparing the WE with Chi speech prior and WCOSH with Chi speech prior estimators, the WE with Chi speech prior estimator often times had slightly better overall performance across the SSNR, PESQ, and SNR Loss objective quality metrics than the WCOSH with Chi speech prior estimator and would be the recommended estimator for filtering noisy signals with more negative values of the weighting law parameter. For future work, the WE and WCOSH estimators would involve further modifications to integrate even more generalized speech prior statistical estimators, namely the generalized Gamma speech prior, to obtain more gains in SSNR, PESQ, and SNR Loss improvements over the traditional Rayleigh speech prior.

\section{References}

Loizou, 2007. P.C. Loizou. Speech Enhancement Theory and Practice. CRC Press (2007)

Ephraim and Malah, 1984. Y. Ephraim, D. Malah. Speech enhancement using a minimum mean-square error short-time spectral amplitude estimator. IEEE Transactions on Acoustics, Speech and Signal Processing, ASSP-32 (1984), pp. 1109-1121

Ephraim and Malah, 1985. Y. Ephraim, D. Malah. Speech enhancement using a minimum mean-square error log-spectral amplitude estimator. IEEE Transactions on Acoustics, Speech and Signal Processing, 33 (1985), pp. 443-445

Loizou, 2005. P.C. Loizou. Speech enhancement based on perceptually motivated Bayesian estimators of the magnitude spectrum. IEEE Transactions on Acoustics, Speech and Signal Processing, 13 (2005), pp. 857869

Andrianakis and White, 2009. I. Andrianakis, P.R. White. Speech spectral amplitude estimators using optimallyshaped gamma and chi priors

Speech Communication, 51 (2009), pp. 1-14

Breithaupt et al., 2008

Breithaupt, C., Krawczyk, M., Martin, R., 2008. Parameterized MMSE spectral magnitude estimation for the enhancement of noisy speech. In: Presented at International Conference on Acoustics, Speech, and, Signal Processing.

Johnson et al., 1994

N. Johnson, S. Kotz, N. BalakrishnanContinuous Univariate Distributions 
(2nd ed.), John Wiley and Sons, New York (1994)

vol. 1

Gray et al., 1980

R.M. Gray, A. Buzo, J.A.H. Gray, Y. MatsuyamaDistortion measures for speech processing

IEEE Transactions on Acoustics, Speech and Signal Processing, ASSP-28 (1980), pp. 367-376

Gradshteyn and Ryzhik, 2007

I.S. Gradshteyn, I.M. RyzhikTables of Integrals, Series, and Products

Academic Press (2007)

Papamichalis, 1987

P.E. PapamichalisPractical Approaches to Speech Coding

Prentice-Hall, New York, NY (1987)

ITU, 2003

ITU, Subjective test methodology for evaluating speech communication systems that include noise suppression algorithm, ITU-T Recommendation, 2003.

Hu and Loizou, 2007

Y. Hu, P.C. LoizouSubjective comparison and evaluation of speech enhancement algorithms

Speech Communication, 49 (2007), pp. 588-601

Hu and Loizou, 2008

Y. Hu, P. LoizouEvaluation of objective quality measures for speech enhancement

IEEE Transactions on Audio, Speech, and Language Processing, 16 (2008), pp. 229-238

Rix et al., 2001

Rix, A., Beerends, J., Hollier, M., Hekstra, A., 2001. Perceptual evaluation of speech quality (PESQ)-A new method for speech quality assessment of telephone networks and codecs. In: Presented at IEEE International Conference of Acoustics, Speech, and, Signal Processing.

Ma and Loizou, 2011

J. Ma, P.C. LoizouSNR loss: a new objective measure for predicting the intelligibility of noise-suppressed speech

Speech Communication, 53 (2011), pp. 340-354

Subcommittee, 1969

I. SubcommitteelEEE recommended practice for speech quality measurements

IEEE Transactions on Audio and Electroacoustics, AU-17 (1969), pp. 225-246

Pearce and Hirsch, 2000

Pearce, D., Hirsch, H.-G., 2000. Performance evaluation of speech recognition systems under noisy conditions. In: Presented at 6th International Conference on Spoken Language Processing (ICSLP), Beijing, China. 